\title{
RESEARCH
}

Open Access

\section{A genomic view of trophic and metabolic diversity in clade-specific Lamellodysidea sponge microbiomes}

Sheila Podell ${ }^{1}$ D, Jessica M. Blanton', Aaron Oliver ${ }^{1}$, Michelle A. Schorn², Vinayak Agarwal ${ }^{3}$, Jason S. Biggs ${ }^{4}$, Bradley S. Moore ${ }^{5,6,7}$ and Eric E. Allen ${ }^{1,5,7,8^{*}}$

\begin{abstract}
Background: Marine sponges and their microbiomes contribute significantly to carbon and nutrient cycling in global reefs, processing and remineralizing dissolved and particulate organic matter. Lamellodysidea herbacea sponges obtain additional energy from abundant photosynthetic Hormoscilla cyanobacterial symbionts, which also produce polybrominated diphenyl ethers (PBDEs) chemically similar to anthropogenic pollutants of environmental concern. Potential contributions of non-Hormoscilla bacteria to Lamellodysidea microbiome metabolism and the synthesis and degradation of additional secondary metabolites are currently unknown.

Results: This study has determined relative abundance, taxonomic novelty, metabolic capacities, and secondary metabolite potential in 21 previously uncharacterized, uncultured Lamellodysidea-associated microbial populations by reconstructing near-complete metagenome-assembled genomes (MAGs) to complement 16S rRNA gene amplicon studies. Microbial community compositions aligned with sponge host subgroup phylogeny in 16 samples from four host clades collected from multiple sites in Guam over a 3-year period, including representatives of Alphaproteobacteria, Gammaproteobacteria, Oligoflexia, and Bacteroidetes as well as Cyanobacteria (Hormoscilla). Unexpectedly, microbiomes from one host clade also included Cyanobacteria from the prolific secondary metabolite-producer genus Prochloron, a common tunicate symbiont.

Two novel Alphaproteobacteria MAGs encoded pathways diagnostic for methylotrophic metabolism as well as type III secretion systems, and have been provisionally assigned to a new order, designated Candidatus Methylospongiales. MAGs from other taxonomic groups encoded light-driven energy production pathways using not only chlorophyll, but also bacteriochlorophyll and proteorhodopsin. Diverse heterotrophic capabilities favoring aerobic versus anaerobic conditions included pathways for degrading chitin, eukaryotic extracellular matrix polymers, phosphonates, dimethylsulfoniopropionate, trimethylamine, and benzoate. Genetic evidence identified an aerobic catabolic pathway for halogenated aromatics that may enable endogenous PBDEs to be used as a carbon and energy source.

\footnotetext{
* Correspondence: eallen@ucsd.edu

'Marine Biology Research Division, Scripps Institution of Oceanography, University of California, San Diego, La Jolla, CA, USA

${ }^{5}$ Center for Marine Biotechnology and Biomedicine, Scripps Institution of Oceanography, University of California, San Diego, La Jolla, CA, USA Full list of author information is available at the end of the article
}

C C The Author(s). 2020 Open Access This article is licensed under a Creative Commons Attribution 4.0 International License, which permits use, sharing, adaptation, distribution and reproduction in any medium or format, as long as you give appropriate credit to the original author(s) and the source, provide a link to the Creative Commons licence, and indicate if changes were made. The images or other third party material in this article are included in the article's Creative Commons licence, unless indicated otherwise in a credit line to the material. If material is not included in the article's Creative Commons licence and your intended use is not permitted by statutory regulation or exceeds the permitted use, you will need to obtain permission directly from the copyright holder. To view a copy of this licence, visit http://creativecommons.org/licenses/by/4.0/ The Creative Commons Public Domain Dedication waiver (http://creativecommons.org/publicdomain/zero/1.0/) applies to the data made available in this article, unless otherwise stated in a credit line to the data. 


\begin{abstract}
(Continued from previous page)
Conclusions: The reconstruction of high-quality MAGs from all microbial taxa comprising greater than $0.1 \%$ of the sponge microbiome enabled species-specific assignment of unique metabolic features that could not have been predicted from taxonomic data alone. This information will promote more representative models of marine invertebrate microbiome contributions to host bioenergetics, the identification of potential new sponge parasites and pathogens based on conserved metabolic and physiological markers, and a better understanding of biosynthetic and degradative pathways for secondary metabolites and halogenated compounds in sponge-associated microbiota.
\end{abstract}

Keywords: Lamellodysidea, Sponge microbiome, Cyanosponge, Hormoscilla, PBDE, Methylospongia, Prochloron

\section{Background}

The prodigious seawater pumping capabilities of filterfeeding marine sponges contribute significantly to carbon cycling in global reef habitats, through the breakdown and remineralization of both dissolved and particulate forms of organic matter [1-3]. Nutrient recycling is facilitated by host-associated microbial communities comprised of diverse sponge-specific taxa [4], including Gamma- and Alphaproteobacteria, Acidobacteria, Actinobacteria, Chloroflexi, Cyanobacteria, Nitrospirae, Tectomicrobia, candidate phylum Poribacteria, and archaeal Thaumarchaeota (reviewed in $[5,6])$.

Some sponge taxa contain abundant cyanobacterial symbionts, whose photosynthetic activities are essential for host health and growth $[7,8]$. Studies measuring potential contributions of photosynthetic symbionts to host energy metabolism have historically used Lamellodysidea herbacea as a prototypical model [8-10]. These studies have suggested that phototrophic cyanobacteria can contribute as much as $80 \%$ of the total sponge carbon budget, although significant variation has been observed among individual sponges [11].

The cyanobacterial symbionts of $L$. herbacea are dominated by a single taxonomic clade known as Hormoscilla spongeliae (historically described under the genus names Oscillatoria and Phormidium), found in filamentous bundles called trichomes that can comprise up to $50 \%$ of sponge weight $[8,12]$. Although Hormoscilla sponge symbionts have never been successfully cultured in the laboratory, two genomes of this bacterial clade have recently been assembled from trichome-enriched metagenomic samples [13]. 16S rRNA gene amplification studies have detected sponge-specific Alpha- and Gammaproteobacteria sequences associated with Lamellodysidea [14], but did not investigate how these nonHormoscilla symbionts might contribute to metabolic activities and ecological interactions in the sponge microbiome.

Hormoscilla sponge symbionts have attracted particular attention for their ability to produce high concentrations of polybrominated diphenyl ethers (PBDEs), which are chemically similar to anthropogenic pollutants of environmental concern [15-19]. Laboratory studies have suggested that naturally occurring PBDEs might act as antimicrobial agents $[20,21]$ or as predator feeding deterrents [22], but the biologically relevant activities of these compounds in native marine habitats remain controversial.

PBDE product diversity among Lamellodysidea sponges is strongly correlated with the taxonomy of both sponge hosts and their Hormoscilla symbionts, suggesting potential co-evolutionary processes linking host genotype with microbiome and metabolome diversity [16]. The discovery that not all sponge-associated Hormoscilla strains produce PBDEs $[15,16]$ offers an opportunity to compare microbial community compositions and metabolic capabilities in PBDE-producing versus non-producing sponge hosts. The current study addresses these questions through metagenomic reconstruction of 21 new sponge-associated microbial metagenome-assembled genomes (MAGs) from Hormoscilla-dominated Lamellodysidea sponge samples, enabling detailed exploration of their potential contributions to the overall microbial community.

\section{Results}

\section{Sponge samples and metagenomic assemblies}

$L$. herbacea sponge samples were collected over a 3-year period from four different coastal locations in Guam, including both summer and winter seasons (Supplementary Table S1, Additional file 1). Based on a phylogenetic tree of internal transcribed spacer (ITS) regions (Fig. 1), these samples included representatives of all four previously described L. herbacea clades [16]. Clade Ia and Ib samples, whose microbial communities have not previously been described, were obtained at multiple different times and locations. Samples from clades II and III, previously analyzed by $16 \mathrm{~S}$ rRNA gene amplification [14], are represented by tissue samples taken from different individual sponges collected on the same dates at a single site.

Assemblies for each metagenomic sample were initially performed using all available reads for that sample (Supplementary Table S2, Additional file 1). Combined properties of read coverage, nucleotide composition, and predicted protein matches to the GenBank nr 


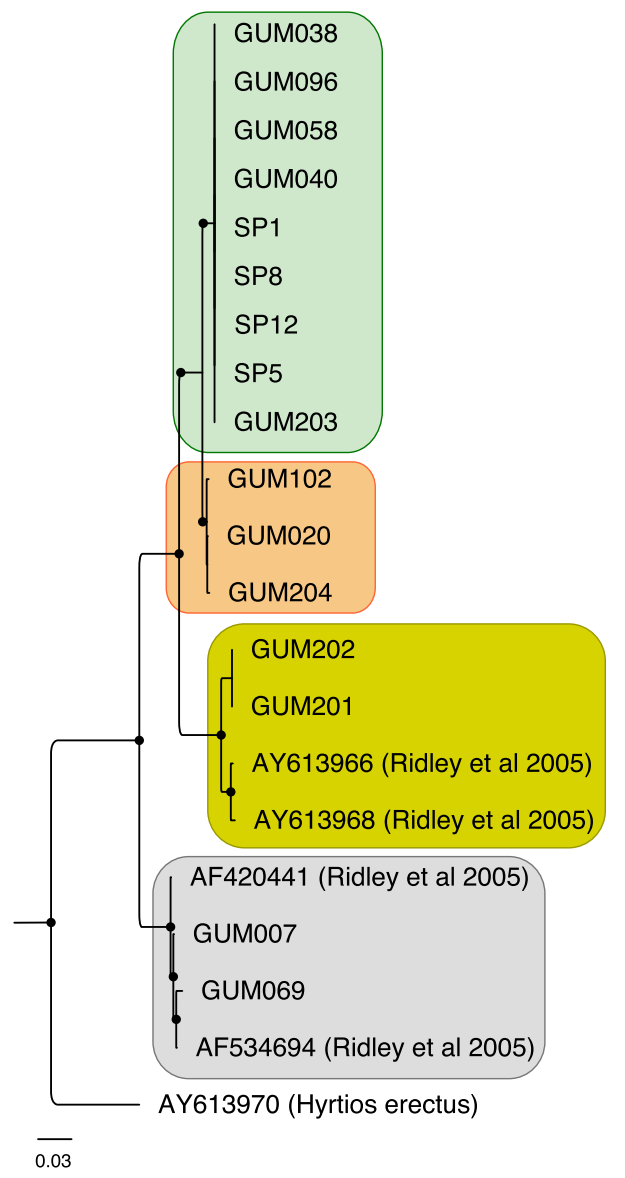

Clade la
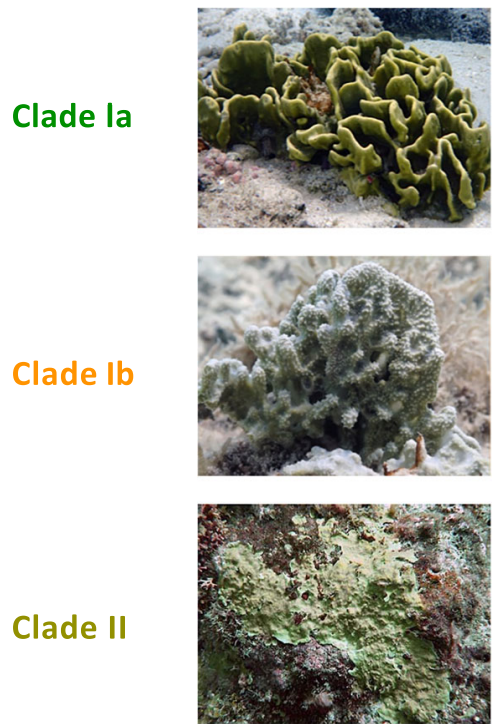

Clade III

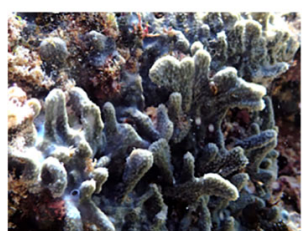

Fig. 1 Phylogenetic tree of Lamellodysidea sponge samples. The tree is based on gene amplification of ITS-2 gene sequences, with Hyrtios erectus as an outgroup. Black dots indicate nodes with bootstrap values exceeding 0.8. Representative samples shown in photographs: clade la, SP5; clade Ib, GUM020; clade II, GUM201; clade III, GUM007

database revealed a relatively small number of discrete scaffold clusters, mapping primarily to database sequences from Cyanobacteria, Bacteroidetes, Alphaproteobacteria, Gammaproteobacteria, and Oligoflexia (Fig. 2). Scaffolds derived from host sponge DNA were present at similar coverage depths to bacterial clusters, but were distinguishable by nucleotide composition and predicted matches to reference database sequences.

Metagenomic scaffold bins were used to recruit matching raw read pairs that were subsequently used in targeted subassemblies to produce 23 consensus population genomes (Table 1; Supplementary Table S3, Additional file 1). All of the assembled genomes except one (clade Ib: GM102ARS1) met or exceeded MIMAG standards for high- or medium-quality drafts [23]. CheckM analysis [24] estimated that 16 of the 23 MAGs were more than $90 \%$ complete, and 19 contained near full-length 16S rRNA gene sequences. The exceptional quality of these MAGs enabled detailed comparisons with both genomically sequenced relatives and environmental 16S rRNA gene surveys.

\section{Taxonomic classification of assembled genomes}

Taxonomic classifications for assembled MAGs were established using concatenated multi-locus phylogenetic trees of conserved proteins and matrices of 16S rRNA gene and average amino acid identity (AAI) scores (Fig. 3; Supplementary Figures S1-5, Additional file 1). AAI scores were especially valuable in providing taxonomic classification levels for genomes with missing or incomplete 16S rRNA genes and those with few sequenced relatives, according to quantitative threshold ranges previously established for large numbers of database examples $[25,26]$.

Cyanobacteria genomes from the genus Hormoscilla were present in all samples, as expected, but sample SP5 from clade Ia also yielded a genome from the genus Prochloron (SP5CPC1; Supplementary Figure S1, Additional file 1). Prochloron are well-known tunicate symbionts [27], but have not previously been discussed in molecular studies of sponge microbiomes. Although Prochloron bacteria were first observed microbiologically on the surfaces of both marine sponges and sea 


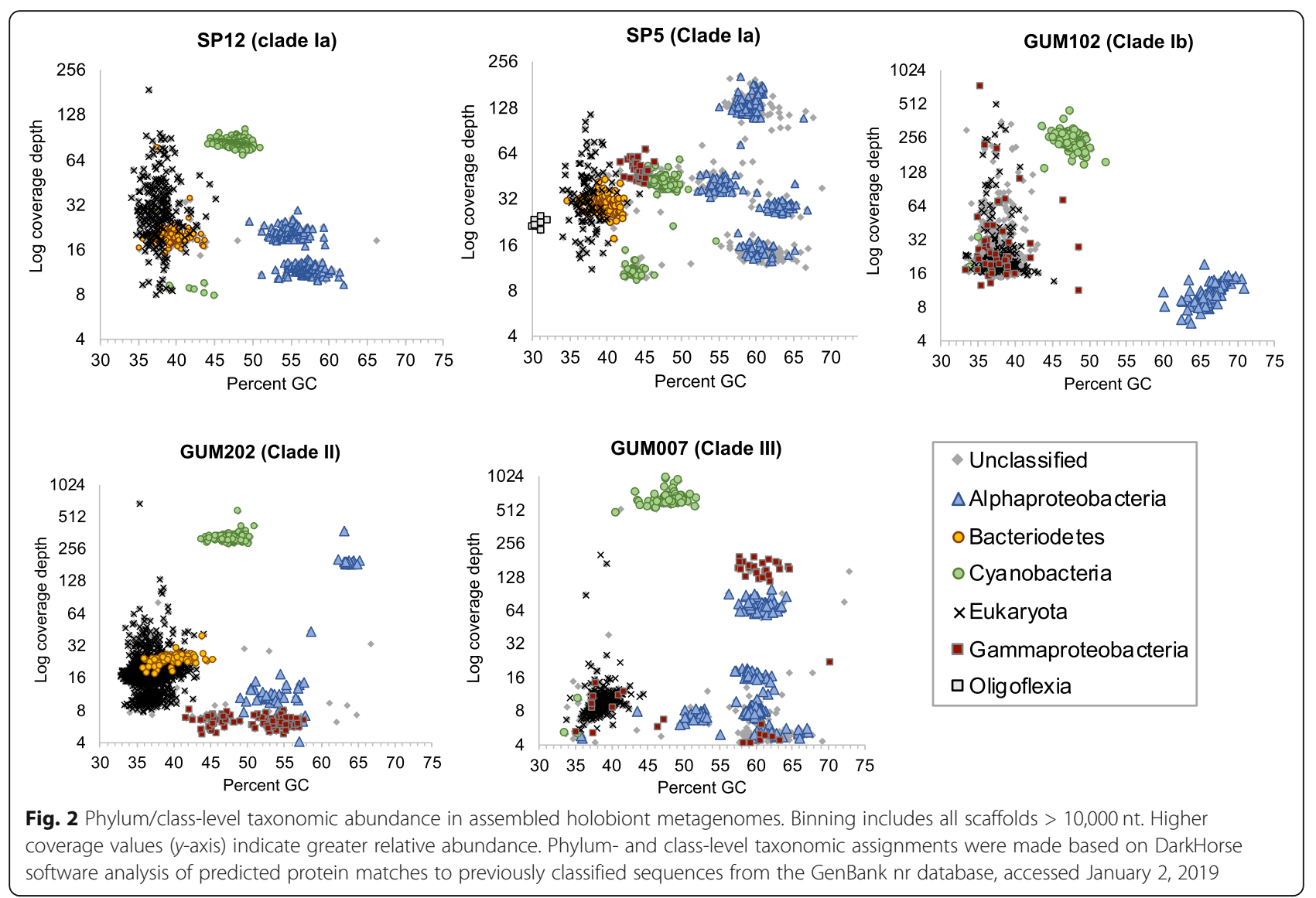

cucumbers more than 30 years ago [28, 29], no spongederived matches to PCR-amplified (Fig. 4) or metagenomically assembled Prochloron 16S rRNA genes from the SP5CPC1 genome were identified at $97 \%$ or greater nucleotide identity in the GenBank $\mathrm{nr}$ database (Supplementary Table S4, Additional file 1) or the Sponge Microbiome Project Database [30, 31] (Supplementary Table S5, Additional file 1). The Prochloron $16 \mathrm{~S}$ rRNA gene sequences obtained in this study do not contain any mismatches to commonly used amplification primers that might explain their absence from these databases, but we are also not aware of any prior $16 \mathrm{~S}$ rRNA gene amplification studies that included Lamellodysidea herbacea samples from clade Ia.

Genomes SP12BCY1, SP5BCY1, and GM202BCY1 were assigned to the Bacteroidetes family Cytophagaceae (Supplementary Figure S2, Additional file 1). Based on AAI scores greater than $98 \%$, these three genomes represent members of the same species. Their closest cultured relative was Ekhidna lutea, a free-living aerobic heterotroph isolated from seawater [32]. 16S rRNA gene identities of $95 \%$ and AAI scores of $67 \%$ suggest membership in the same genus as E. lutea.

Four Alphaproteobacteria genomes (SP12ARB1, SP5ARB1, GM7ARB1, GM7ARB2) were classified in family Rhodobacteraceae (Supplementary Figure S3, Additional file 1). Their most closely related cultured isolate was Nioella sediminis, a free-living aerobic marine bacterium from a sister genus of the Roseobacteria clade [33, 34]. Alphaproteobacteria genomes SP5ARS3, GM102ARS1, and GM202ARS1 fell within family Rhodospirillaceae. Terrestrial plant-associated Azospirillum brasilense [35] was their closest sequenced relative. Other members of genus Azospirillum have been identified in environmental samples from marine habitats, but not yet cultured or sequenced. An AAI score of $87 \%$ suggests that SP5ARS3 should be classified in the genus Azospirillum, but GM102ARS1 and GM202ARS1, with AAI scores of $49-51 \%$, represent a new genus within the family Rhodospirillaceae. Alphaproteobacterial genomes SP12AHP2 and SP5AHP1 were classified in the Hyphomonadaceae family, as a sister genus to closest isolate relative Hellea balneolensis, an aerobic, heterotrophic bacterium isolated from surface seawater [36]. SP12AHP2 and SP5AHP1 have identical $16 \mathrm{~S}$ rRNA genes and AAI scores of $99 \%$, qualifying them as members of the same species in a new genus of family Hyphomonadaceae (Supplementary Figure 2B, Additional file 1). 
Table 1 Metagenome-assembled genome properties

\begin{tabular}{|c|c|c|c|c|c|c|c|c|c|}
\hline $\begin{array}{l}\text { Host } \\
\text { clade }\end{array}$ & MAG name & $\begin{array}{l}\text { Total } \\
\text { length }\end{array}$ & $\begin{array}{l}\mathrm{PCt} \\
\mathrm{GC}\end{array}$ & $\begin{array}{l}\text { Num } \\
\text { scf }\end{array}$ & $\begin{array}{l}\text { Gene } \\
\text { count }\end{array}$ & $\begin{array}{l}16 S \\
\text { len }\end{array}$ & $\begin{array}{l}\text { CheckM } \\
\text { complete }\end{array}$ & $\begin{array}{l}\text { CheckM } \\
\text { contam }\end{array}$ & Taxonomy \\
\hline la & SP12AHP1 & $\begin{array}{l}2,316 \\
397\end{array}$ & 54.6 & 125 & 2323 & 1467 & 91.4 & 0.0 & $\begin{array}{l}\text { Alphaproteobacteria; Rhodobacterales; } \\
\text { Hyphomonadaceae }\end{array}$ \\
\hline la & SP12ARB1 & $\begin{array}{l}2,913 \\
125\end{array}$ & 57.2 & 262 & 3307 & 1381 & 69.1 & 0.0 & $\begin{array}{l}\text { Alphaproteobacteria; Rhodobacterales; } \\
\text { Rhodobacteraceae }\end{array}$ \\
\hline la & SP12BCY1 & $\begin{array}{l}2,978 \\
903\end{array}$ & 39.9 & 202 & 2868 & 1517 & 100.0 & 0.0 & $\begin{array}{l}\text { Bacteroidetes; Cytophagia; Cytophagales; } \\
\text { Cytophagaceae; Ekhidna }\end{array}$ \\
\hline la & SP12CHS1 & $\begin{array}{l}4,999 \\
041\end{array}$ & 48.0 & 573 & 6155 & 1486 & 96.6 & 0.2 & $\begin{array}{l}\text { Cyanobacteria; Oscillatoriophycideae; Oscillatoriales; } \\
\text { Gomontiellaceae; Hormoscilla }\end{array}$ \\
\hline la & SP5AHP2 & $\begin{array}{l}2,126 \\
940\end{array}$ & 54.9 & 153 & 2340 & 1467 & 87.8 & 0.0 & $\begin{array}{l}\text { Alphaproteobacteria; Rhodobacterales; } \\
\text { Hyphomonadaceae }\end{array}$ \\
\hline la & SP5ARB1 & $\begin{array}{l}2,540 \\
908\end{array}$ & 59.8 & 118 & 2682 & 1415 & 82.4 & 3.4 & $\begin{array}{l}\text { Alphaproteobacteria; Rhodobacterales; } \\
\text { Rhodobacteraceae }\end{array}$ \\
\hline la & SP5ARS3 & $\begin{array}{l}2,817 \\
712\end{array}$ & 63.5 & 46 & 2617 & n.d & 94.8 & 0.0 & Alphaproteobacteria: Rhodospirillales \\
\hline la & SP5BCY1 & $\begin{array}{l}2,936 \\
924\end{array}$ & 39.7 & 200 & 2813 & 1517 & 96.4 & 0.5 & $\begin{array}{l}\text { Bacteroidetes; Cytophagia; Cytophagales; } \\
\text { Cytophagaceae; Ekhidna }\end{array}$ \\
\hline la & SP5CHS1 & $\begin{array}{l}4,838 \\
078\end{array}$ & 47.7 & 445 & 5922 & 1484 & 96.6 & 0.2 & $\begin{array}{l}\text { Cyanobacteria; Oscillatoriophycideae; Oscillatoriales; } \\
\text { Gomontiellaceae; Hormoscilla }\end{array}$ \\
\hline la & SP5CPC & $\begin{array}{l}2,787 \\
143\end{array}$ & 43.8 & 397 & 3056 & 1442 & 77.4 & 0.0 & $\begin{array}{l}\text { Cyanobacteria; Synechococcales; Prochloraceae; } \\
\text { Prochloron }\end{array}$ \\
\hline la & SP5GCR1 & $\begin{array}{l}1,621 \\
865\end{array}$ & 44.7 & 28 & 1518 & 1542 & 98.3 & 0.0 & Gammaproteobacteria; Chromatiales \\
\hline la & SP5OBV1 & $\begin{array}{l}1,589 \\
243\end{array}$ & 29.8 & 57 & 1544 & 1469 & 91.2 & 0.0 & Oligoflexia; Bdellovibrionales \\
\hline $\mathrm{lb}$ & GM102ARS1 & $\begin{array}{l}1,092 \\
149\end{array}$ & 65.2 & 302 & 1180 & 263 & 41.2 & 0.8 & Alphaproteobacteria: Rhodospirillales \\
\hline $\mathrm{lb}$ & GM102HS1 & $\begin{array}{l}5,041 \\
890\end{array}$ & 47.5 & 518 & 6591 & 227 & 95.7 & 1.8 & $\begin{array}{l}\text { Cyanobacteria; Oscillatoriophycideae; Oscillatoriales; } \\
\text { Gomontiellaceae; Hormoscilla }\end{array}$ \\
\hline$\|$ & GM202ARS1 & $\begin{array}{l}2,820 \\
038\end{array}$ & 63.5 & 18 & 2912 & 1500 & 100.0 & 0.0 & Alphaproteobacteria: Rhodospirillales \\
\hline$\|$ & GM202ARS2 & $\begin{array}{l}1,828 \\
912\end{array}$ & 53.3 & 49 & 1885 & 1496 & 85.3 & 0.8 & Alphaproteobacteria: Candidatus Methylospongiales \\
\hline$\|$ & GM202BCY1 & $\begin{array}{l}3,008 \\
284\end{array}$ & 40.3 & 122 & 2918 & 1517 & 94.9 & 0.5 & $\begin{array}{l}\text { Bacteroidetes; Cytophagia; Cytophagales; } \\
\text { Cytophagaceae; Ekhidna }\end{array}$ \\
\hline$\|$ & GM2O2CHS1 ${ }^{a}$ & $\begin{array}{l}6,869 \\
623\end{array}$ & 47.5 & 70 & 7441 & 1492 & 97.4 & 2.0 & $\begin{array}{l}\text { Cyanobacteria; Oscillatoriophycideae; Oscillatoriales; } \\
\text { Gomontiellaceae; Hormoscilla }\end{array}$ \\
\hline III & GM7ARB1 & $\begin{array}{l}2,553 \\
113\end{array}$ & 60.7 & 59 & 2499 & 1463 & 96.6 & 0.0 & $\begin{array}{l}\text { Alphaproteobacteria; Rhodobacterales; } \\
\text { Rhodobacteraceae }\end{array}$ \\
\hline III & GM7ARB2 & $\begin{array}{l}2,578 \\
993\end{array}$ & 59.9 & 37 & 2551 & 1235 & 93.7 & 0.9 & $\begin{array}{l}\text { Alphaproteobacteria; Rhodobacterales; } \\
\text { Rhodobacteraceae }\end{array}$ \\
\hline III & GM7ARS4 & $\begin{array}{l}1,589 \\
243\end{array}$ & 52.0 & 24 & 1425 & 66 & 91.0 & 0.8 & Alphaproteobacteria: Candidatus Methylospongiales \\
\hline III & $\mathrm{GM} 7 \mathrm{CHS1}{ }^{\mathrm{b}}$ & $\begin{array}{l}6,299 \\
679\end{array}$ & 47.8 & 6 & 6160 & 1484 & 100.0 & 1.8 & $\begin{array}{l}\text { Cyanobacteria; Oscillatoriophycideae; Oscillatoriales; } \\
\text { Gomontiellaceae }\end{array}$ \\
\hline III & GM7GCV1 & $\begin{array}{l}1,911 \\
341\end{array}$ & 61.0 & 40 & 1792 & 1524 & 89.1 & 0.0 & Gammaproteobacteria; Cellvibrionales \\
\hline
\end{tabular}

Additional metadata and genome quality information are provided in Supplementary Table 3; n.d. not detected. Symbols denote genomes previously described in [13]

${ }^{a}$ MAG GUM202CHS1 is identical to NCBI accession RFFC00000000.1

${ }^{\mathrm{b}}$ MAG GM7CHS1 was newly assembled as described in the "Methods" section, replacing NCBI accession RFFB00000000.1 


\section{Bacteroidetes}

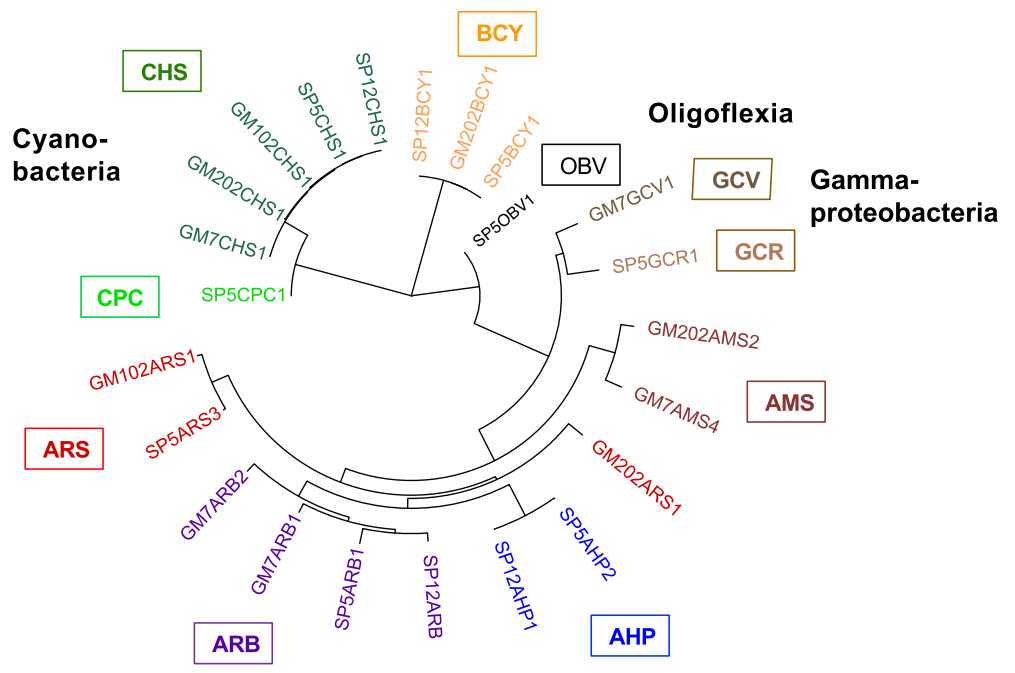

Alphaproteobacteria

Fig. 3 Concatenated multi-locus PhyloPhIAn tree of assembled MAG taxonomic diversity. Assembled genome group name abbreviations, in clockwise order: BCY, Bacteroidetes Cytophagaceae; OBV, Oligoflexia Bdellovibrionales; GCV, Gammaproteobacteria Cellvibrionales; GCR, Gammaproteobacteria Chromatiales; AMS, Alphaproteobacteria Methylospongia; AHP, Alphaproteobacteria Hyphomonadaceae; ARB, Alphaproteobacteria Rhodobacteraceae; ARS, Alphaproteobacteria Rhodospirillales; CPC, Cyanobacteria Prochloron; CHS, Cyanobacteria Hormoscilla. More detailed trees for each phylum-level group are provided in Supplementary Figures 1-5

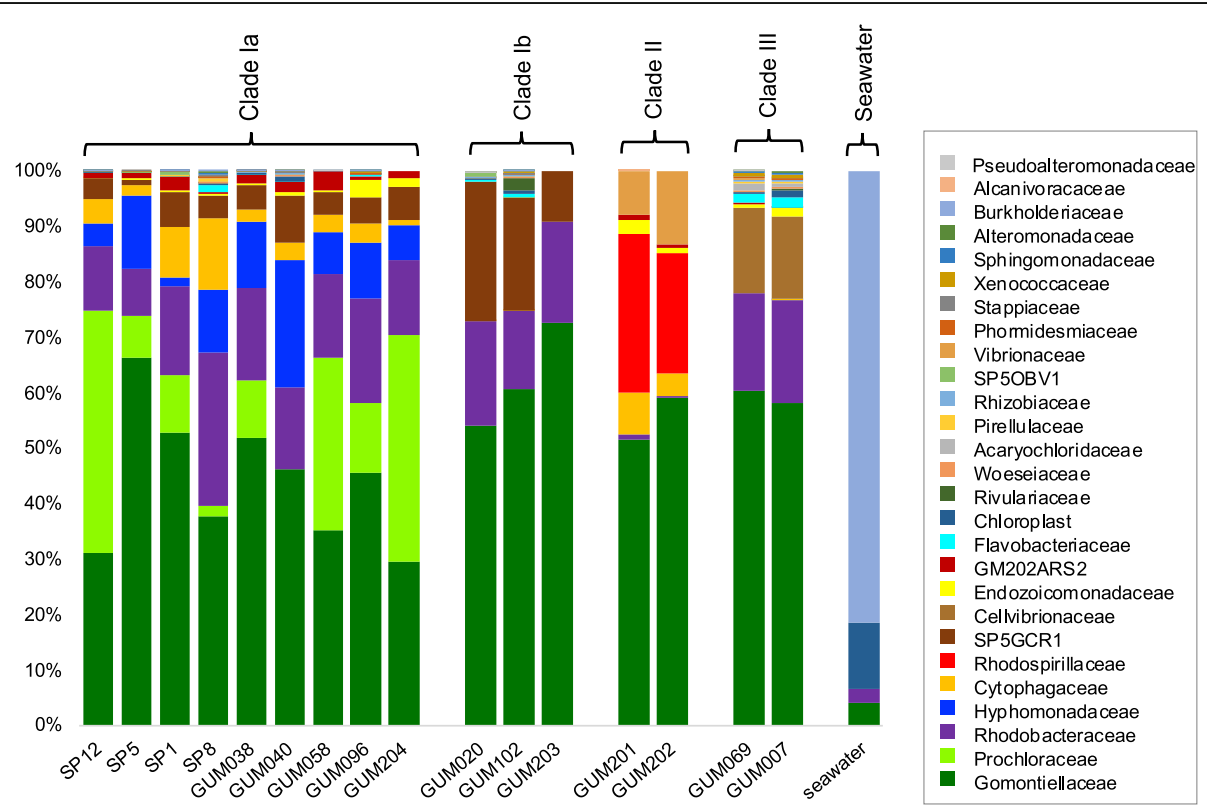

Fig. 4 Relative abundance of family-level taxonomic groups. Based on blastn matches to $16 \mathrm{~S}$ rRNA gene sequences in the SILVA reference database, version 132. Seawater values were obtained from sample GUM061 (Supplementary Table 1). The GM102ARS1, GM102CHS1, SP5ARS3, and GM7ARS4 MAGs did not include any sequences containing the primer-amplified 16S rRNA gene region, so these taxa are not included in this analysis. Relative abundances of these sequences, based on Bowtie2 recruitment of metagenomic reads, are shown in Supplementary Figure 7 
GM7GCV1 was the only genome sequenced from the Gammaproteobacteria order Cellovibrionales (Supplementary Figure 4, Additional file 1). Its closest sequenced relative, Halieaceae LZ-16-2, is an uncharacterized bacterium obtained from a mixed laboratory culture with the saxitoxin-producing dinoflagellate Alexandrium tamarense (NZ_RFLW00000000.1). AAI scores of 50-65\% with other members of family Halieaceae suggest GM7GCV1 might be classified as a new genus within this family, but 16S rRNA gene identities of $88-89 \%$ imply a more distant relationship, potentially in a new family.

The remaining community genomes were too distant from reference database examples to allow precise taxonomic assignments. GM7GCR1 had no close matches among sequenced isolates, being most closely related to unclassified environmental Gammaproteobacteria, with 16S rRNA gene nucleotide sequence identities of $90 \%$ and AAI scores below 45\% (Supplementary Figure 3 and Supplementary Tables S4-5, Additional file 1). These results suggest it should be classified as either a new family within Chromatiales or a new order within Gammaproteobacteria.

Two Alphaproteobacteria genomes (GM202ARS2, GM7ARS4) fell outside any previously established orders, and could only be assigned at the class level, although their 55\% AAI value when compared to each other implies they might be members of a single family (Supplementary Figure S2, Additional file 1). Genome SP5OBV1 was most closely related to Bdellovibrio bacteriovorus from the order Bdellovibrionales, but low 16S rRNA gene identity (82\%) and AAI (40\%) scores suggest it might represent a new, previously unreported order within the recently described Proteobacteria class Oligoflexia (Supplementary Figure S5, Additional file 1) [37]. Verification of these taxonomic assignments will require the discovery of additional sequences for closely related taxa.

16S rRNA genes from Rhodobacteriaceae and Rhodospirillaceae genomes were conserved in samples obtained over 3 years in the current study, and also matched previously reported sequences from a 2005 amplification study of Guam Lamellodysidea sponges [14] at 98-100\% identity (Supplementary Table S4, Additional file 1). No 16S rRNA gene matches to the Cytophagaceae, Cellvibrionales, Hyphomonadaceae, and Bdellovibrionales genomes from this study were detected in the 2005 Lamellodysidea microbiome study, but this may be due to the absence of samples from host clades Ia and Ib and the small number of clones analyzed ( $<40$ per sample) in this earlier work [14]. The closest GenBank 16S rRNA gene matches to MAGs in the current study were associated with Ircinia, Tethya. Stylissa, and Axinella sponges, at $90-96 \%$ identity levels (Supplementary Table S4,
Additional file 1). Additional low abundance 16S rRNA gene matches were present at $97 \%$ identity in numerous other sponge genera from the Sponge Microbiome Project Database (Supplementary Table S5, Additional file 1). No species-level matches were detected in this database for 16S rRNA gene sequences from GM7GVC1 or SP5CPC1, but more distant matches were found at $95 \%$ identity, suggesting the presence of bacteria from the same genera but not the same species [38].

\section{Relative abundance comparisons between samples}

Microbial community compositions in all samples were strongly correlated with host clade taxonomy (Fig. 4). The most taxonomically diverse communities were observed in host clade Ia, which included two bacterial taxa that were absent from all other clades: alphaproteobacterial family Hyphomonadaecae and cyanobacterial genus Prochloron. Host clade II samples were notable for the consistent replacement of Alphaproteobacteria family Rhodobacteraceae with Rhodospirillaceae. Sponge metagenome 16S rRNA gene sequences from assembled MAGs accounted for $44-88 \%$ of all bacterial amplicon sequence variants (ASVs) in sponge samples, but only $0.1 \%$ in nearby seawater (Supplementary Figure S6, Additional file 1).

Relative abundances of the four MAGs with incomplete or missing 16S rRNA sequences (GM102ARS1, GM102CHS1, SP5ARS3, and GM7ARS4) were assessed by recruitment of unassembled metagenomic reads to assembled MAGs (Supplementary Figure S7, Additional file 1). Metagenomic read recruitment is known to underestimate relative abundance of genomes with smaller sizes, but 16S rRNA gene amplification overreports taxa with multiple gene copies, precluding exact numerical agreement. Despite these differences, results from both procedures were consistent in showing the host clade-specificity of taxonomic compositions, including greater diversity and the unique presence of Prochloron and Hyphomonadaceae in clade 1a, as well as the replacement of Rhodobacteraceae with Rhodospirillaceae in clade II samples. These patterns were consistent over all available sample collection time points and locations tested.

\section{Predicted bacterial lifestyles}

Predicted protein annotations for the 23 microbial genomes in this study were used to explore both broad, community-wide patterns and detailed metabolic pathways specific to individual genomes (Fig. 5). Shared features among all taxonomic groups included the presence of aerobic respiration, glycolysis, and TCA cycle enzymes. None of the assembled genomes contained genes encoding flagellar biosynthesis, although genes for gliding motility were found in all 


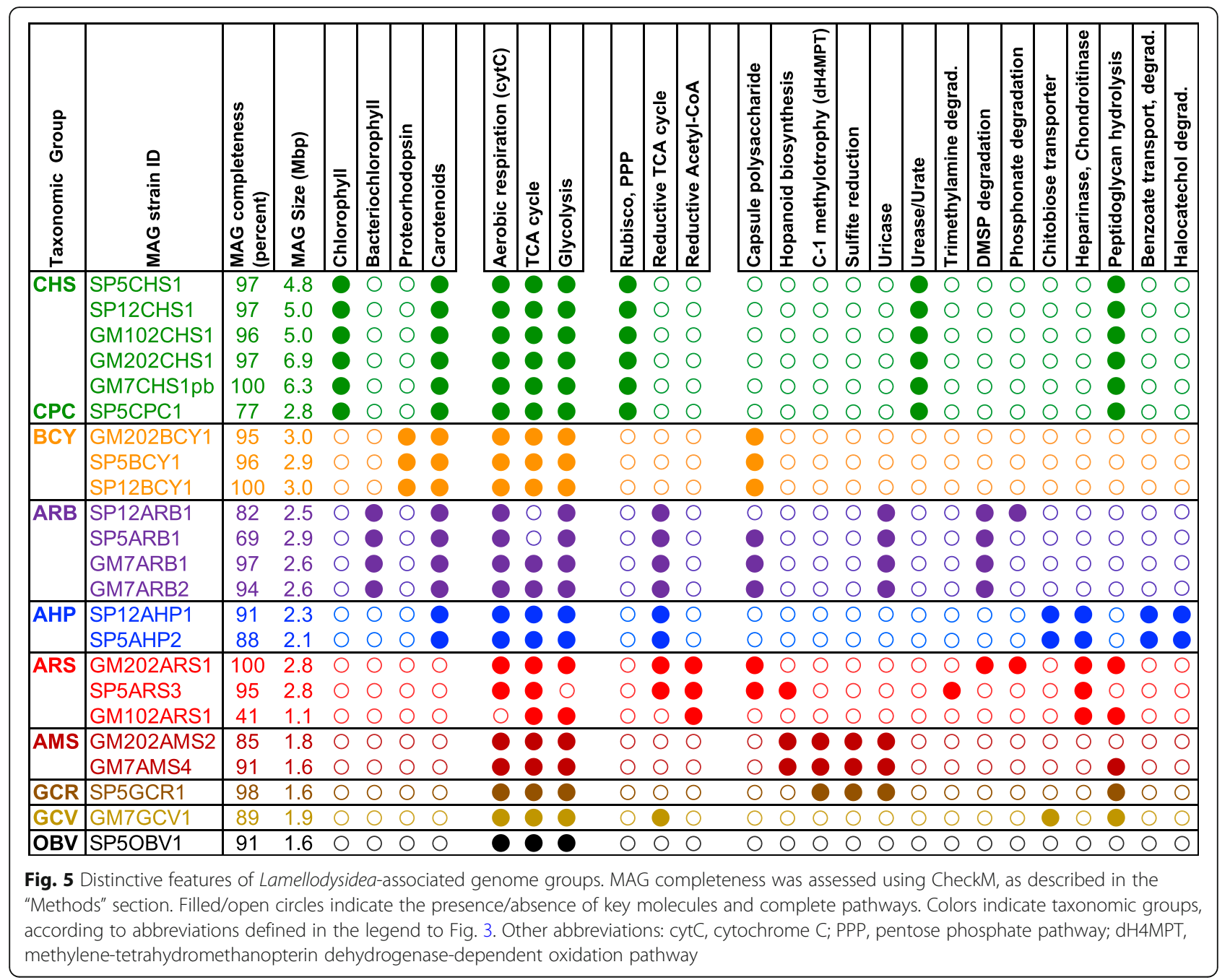

Bacteroidetes family Cytophagaceae genomes (group $\mathrm{BCY}$ ), and twitching motility in Cyanobacteria (groups $\mathrm{CHS}$ and $\mathrm{CPC}$ ), one of the Gammaproteobacteria (group GCV), and Oligoflexia (group OBV) genomes (Supplementary Table S6, Additional file 1). Several genome groups contained expanded gene families encoding adhesive molecules with the potential to resist shear forces from high seawater flow rates, for example genes encoding cadherin and ankyrin domains in all Bacteroidetes family Cytophagaceae (BCY) and Gammaproteobacteria group GCR genomes, as well as type IV pilus structures in Cyanobacteria, Alphaproteobacteria Candidatus Methylospongiales, Gammaproteobacteria, and Oligoflexia genomes (groups CHS, CPC, AMS, GCR, GCV, and OBV). The absence of nitrogenase complex genes in any of the assembled MAGs, combined with the near-universal presence of ammonia transporters, suggests ready availability of fixed nitrogen, consistent with detection of ammonia excretion in many sponge species [2].
Metabolic phenotype analysis of the assembled genomes identified pathways associated with phototrophic, methylotrophic, heterotrophic, and parasitic or pathogenic lifestyles. Phototrophic pathways included not only chlorophyll-based photosynthesis with carbon fixation via the Calvin-Benson cycle in Hormoscilla and Prochloron cyanobacterial genomes, but also bacteriochlorophyllmediated anoxygenic photosynthesis in alphaproteobacterial Rhodobacteriaceae, coupled with carbon fixation via the reductive TCA cycle. A third mode of light-driven energy production was identified in Bacteroidetes family Cytophagaceae genomes, supporting ATP synthesis through proteorhodopsin-generated proton motive force. Rhodobacteriaceae, Hyphomonadaceae, and Cytophagaceae genomes all contained carotenoid biosynthesis pathways, offering potential protection against free radicals generated during photosynthesis and/or exposure to ultraviolet radiation.

The GM7ARS4, GM202ARS2, and SP5GCR1 genomes all included a complete methylene-tetrahydromethanopterin 
dehydrogenase (dH4MPT)-dependent oxidation pathway, diagnostic for methylotrophic C-1 metabolism [39]. Each of these genomes also encoded a complete type III secretion system (T3SS), often associated with eukaryotic pathogenicity. No pathways for dH4MPT-dependent oxidation or T3SS biosynthesis were present in other genomes recovered from this study. Based on these data, the taxonomic relationship between GM7ARS4 and GM202ARS2, and the absence of any other previously described bacteria from the same order, we have provisionally assigned these two genomes to a new order named Candidatus Methylospongiales.

T3SS operons in both SP5GCR1 and the two Methylospongiales genomes contained $15-18$ proteins annotated as T3SS components, including base, inner rod, and needle proteins; pore-forming translocation proteins; chaperonins; ATPases; and regulatory proteins. Although it was not possible to determine the nature of substrates being transported, T3SS operons also included matches to distinctive virulence-associated protein families (e.g., YscX), associated with human pathogens [40]. Placement of sponge microbiome YscR homologs in a reference tree of conserved, habitatclassified examples (Supplementary Figure S8, Additional file 1) [41] shows their closest relatives derive from extracellular bacteria associated with animal and insect hosts, but not plants, protists, or fungi. These results suggest possible roles for GM7ARS4, GM202ARS2, and SP5GCR1 in sponge host parasitism and/or pathogenicity. Genome sizes ranged from 1.6 to $1.8 \mathrm{Mbp}$, consistent with small sizes often observed in obligate symbionts and pathogenic bacteria [42-44].

Evidence identifying other community members as potential obligate symbionts was constrained by assembled genome incompleteness and limited availability of wellcharacterized free-living relatives. However, supporting evidence was provided in some cases by consistent presence or absence of clade-specific diagnostic features in multiple closely related MAGs from different samples. As an example, Hormoscilla genomes GUM202 and GUM007 have previously been shown to lack complete pathways for biotin synthesis [13]. This same deficiency was confirmed in Hormoscilla genomes from samples SP5, SP12, and GUM102, along with the presence of multiple transporters for importing this essential cofactor.

Genomic streamlining has previously been proposed as a shared feature of pelagic marine Rhodbacteriacae of the Roseobacter clade [45]. The genomes of GM7ARB1 and GM7ARB2, estimated to be $94-97 \%$ complete by CheckM analysis, are approximately $2.55 \mathrm{Mbp}$ in size. This is smaller than the previously reported minimum genome size of $3.3 \mathrm{Mbp}$ for Roseobacters, as well as closest free-living relative Nioella nitratereducens (4.0
Mbp). The four Rhodbacteriacae in the current study have $8-10 \%$ of their genomes devoted to transporter functions (Supplementary Table S6, Additional file 1), but lack genes for flagellar biosynthesis, and RuBisCOmediated carbon fixation typically found in other family members. However, many other clade-specific characteristics have been preserved, including pathways for the synthesis of capsular polysaccharides and the degradation of phosphonates, urate, and dimethylsulfoniopropionate (DSMP).

Marine Bacteroidetes from the CytophagaFlavobacteria group are noted for their role in degrading organic matter during phytoplankton blooms, but group members containing proteorhodopsin have consistently smaller genomes than close taxonomic relatives lacking this gene function [46]. Consistent with these observations, Cytophagaceae genomes SP12BCY2, SP5BCY1, and GM202BCY1 ( $3 \mathrm{Mbp})$ are similar in size to freeliving family members containing proteorhodopsin, but smaller than closest sequenced relative Ekhidna lutea (4.2 Mbp), which does not. SP12BCY2, SP5BCY1, and GM202BCY1 are enriched in signal peptidecontaining peptidases (9-12 per genome) and glycosidases (3-4 per genome), suggesting the retention of conserved heterotrophic capabilities for degrading extracellular proteins and polysaccharides (Supplementary Table S6, Additional file 1).

All three of these genomes have retained Bacteroidetes-specific gliding motility and Por (type IX) secretion system functions. The type IX secretion system is both an essential component of gliding motility in non-pathogenic species $[47,48]$ and a virulence factor in human and fish pathogens [49-51]. A large number of novel cadherin domain-containing proteins (Supplementary Table S6, Additional file 1), with closest GenBank matches at $29-44 \%$ amino acid identity, may facilitate adhesion to sponge hosts and/or particulate matter [52].

Potential symbiotic adaptations in Hyphomonadaceae genomes SP12HP1 and SP5AHP2 were suggested by the absence of both flagellar motility genes and the stalk formation pathway found in many other Hyphomonadaceae genomes [53]. However, genome sizes (2.4-2.5 Mbp) were only about $25 \%$ smaller than closest free-living relative Hellea balneolensis (3.2 Mbp). Lineage characteristic genes for heparinases, chondroitinases, and chitinases, and signal peptide-containing glycosidases were retained in SP12HP1 and SP5AHP2, potentially facilitating the degradation of host extracellular matrix as well as organic marine particulates. The presence of typical Hyphomonadaceae pathways for the transport and degradation of aromatic compounds such as benzoic acid suggests retention of diverse heterotrophic capabilities.

Rhodospirillales genomes SP5ARS3, GUM102ARS1, and GM202ARS1 have much smaller genomes than their 
closest sequenced relative, the terrestrial soil bacterium Azospirillum brasilense ( $<3$ versus $7.2 \mathrm{Mbp}$ ), but this difference could be due to expansion in Azospirillum rather than reduction in sponge-associated genomes. SP5ARS3, GUM102ARS1, and GM202ARS1 are unique among the genomes of this study in encoding pathways for carbon fixation via the Wood-Ljungdahl (Reductive Acetyl-CoA) pathway, the glyoxylate cycle, and trimethylamine degradation. The presence of nitrile hydratases and a large repertoire of transporters $(9-12 \%$ of predicted coding sequences; Supplementary Table S6, Additional file 1) with predicted specificities for amino acids, oligopeptides, taurine, spermidine/putrescein, lipoproteins, ribose/xylose, and glycerol suggest metabolic versatility encompassing a wide variety of substrates, especially those containing amine groups. Genes encoding capsular biosynthesis may provide protection against host phagocytosis, viral attack, and/or hydrophobic toxins.

Marine relatives of genome GM7GCV1 include both free-living and host-associated species from Gammaproteobacteria family Cellovibronaceae, with AAI scores of $50 \%$ and $16 \mathrm{~S}$ rRNA gene identities of $87-88 \%$. All cultured examples have genome sizes of $4 \mathrm{Mbp}$ or larger, including the dinoflagellate-associated Halieaceae LZ16-2, GM7GCV1's closest relative. The much smaller genome size of GM7GCV (1.8 Mbp) is typical for more distantly related, marine particle-associated Cellovibronaceae from the Tara Oceans project (e.g., TMED119 at AAI 45\%; Supplementary Figure S3, Additional file 1), whose genome sizes range from 1.1 to $2.9 \mathrm{Mbp}$ [54]. Factors responsible for smaller genome sizes in these uncultured, uncharacterized bacterial relatives are unknown.

The unavailability of sequenced genomes for relatives closer than order level makes it difficult to determine whether the SP5OBV1 genome lacks clade-specific features. However, it is unlikely that this species follows the same bacterivorous lifestyle as its distant cousins, not only because its genome size, estimated at $91 \%$ complete by CheckM analysis, is less than half as large as closest relative Bdellovibrio bacteriovorus (1.7 versus $3.8 \mathrm{Mbp}$ ), but also due to the absence of genes encoding flagellar motility and proteoglycan synthesis described as essential for this activity in other Bdellovibrionales species (reviewed in [55]). Fatty acid auxotrophy in SP5OBV1 is suggested by the absence of acetyl-CoA carboxylase and all other fatty acid biosynthesis pathway enzymes except FabG, coupled with the presence of complete pathways for lipoic acid metabolism and fatty acid degradation. Some mammalian pathogens are known to suppress endogenous biosynthesis while incorporating exogenous host fatty acids as a triclosan resistance mechanism, but previously reported genomic pathway deficiencies accompanying these adaptations have been much less extensive than those observed in SP5OVB1 [56].

\section{Viral sequences and phage defense}

Electron microscopic studies have identified phage-like particles associated with Lamellodysidea sponge samples [57], but these have not yet been characterized by sequence analysis. In this study, viral scaffold candidates detected by VirSorter [58] in assembled holobiont metagenomes were dominated by GenBank matches to double stranded DNA tailed bacteriophage (Caudovirales) of the Siphoviridae, Myoviridae, and Podoviridae lineages (Supplementary Figure S9, Additional file 1). Matches to Microviridae, archaeal Bicaudaviridae, and eukaryotic Baculoviridae and Herpes viruses were also detected at low levels. Genetic heterogeneity of integrated phage regions can make them difficult to capture in metagenomic assemblies, potentially underestimating occurrence. However, abundant CRISPR sequences, transposons, and restriction enzymes roughly proportional to genome size in assembled MAGs suggest a past history of recurring viral challenges (Supplementary Table S7, Additional file 1).

Integrated prophage genomes sometimes carry passenger genes of bacterial origin that can modify the phenotype of the host, resulting in improved fitness of infected cells, a process known as lysogenic conversion [59]. Lamellodysidea-associated phage candidate scaffolds included bacterial genes encoding DNA methyltransferases, glucanases, peptidases, and vitamin B-12 biosynthesis. One candidate Myoviridae scaffold, from sample SP5, encoded two key enzymes (PhnI and PhnJ) of the carbon-phosphonate lyase pathway [60]. This pathway has previously been demonstrated to be enriched under phosphate limiting conditions in metagenomic marine microbes [61], and could potentially assist in liberating phosphate from recalcitrant organic particles.

\section{Secondary metabolite pathways}

Secondary metabolite gene cluster candidates identified by antiSMASH [62] were most abundant in cyanobacterial taxa, with the largest number in previously described Hormoscilla genomes GM7CHS1 and GM202CHS1 (Supplementary Table S8, Additional file 1 [13];). Hormoscilla genomes SP12CHS1, SP5CHS1, and GM102CHS1 had fewer predicted clusters, but antiSMASH cluster detection sensitivity may have been reduced by scaffold fragmentation in these MAGs, which were assembled from Illumina reads without PacBio read supplementation.

The next most abundant source of biosynthetic gene clusters was Prochloron genome SP5CPC1, predicted to include two non-ribosomal peptide synthetases (NRPS), four terpene synthases, three ribosomally synthesized 
post-translationally modified peptides (RiPPs), and two flavin-dependent aromatic halogenases (Supplementary Table S8, Additional file 1). Although SP5CPC1 has a smaller genome size with fewer total clusters than previously sequenced Prochloron relatives [63], SP5CPC1 is also less complete, with short scaffolds and fragmented, incomplete pathway sequences that cannot easily be linked to specific molecular products. The aromatic halogenases were unrelated to those found in PBDEproducing strains of Hormoscilla spongeliae ([16], Supplementary Table S10B, Additional file 1), and putative RiPP clusters could only be classified as distantly related to non-cyanobactin bacteriocins and lassopeptides, based on HMM (Hidden Markov Model) pattern matches [62]. None of the putative RiPP clusters contained genes from the well-characterized patellamide pathway of Prochloron didimei [64].

Predicted biosynthetic clusters in non-cyanobacterial genomes were limited to terpenes and bacteriocinrelated RiPPs, except for the SP12BCY1, SP5BCY1, and GUM202BCY1 genomes, which all contained an identical type III polyketide synthase (PKS). The closest database matches to this protein were from Cytophagaceae genera such as Ekhidna, Marinoscillum, and Pontibacter at 53-64\% amino acid identity. Biosynthesis of plant-like flavonoids like those recently characterized in Flavibacteria cheonhonense [65] seems unlikely, because key pathway enzyme phenylalanine ammonia lyase is missing $[66,67]$. However, type III PKS genes from the current study also matched CepAB genes from taxonomically distant sponge symbiont Entotheonella gemina (class Tectomicrobia) at $48 \%$ amino acid identity, suggesting phenolic lipids as a potential biosynthetic product [68].

Previously reported gene clusters encoding polybrominated compounds in assembled Hormoscilla genomes [13, 16] do not account for the full range of halogenated products described in Lamellodysidea sponges [69]. Additional diversity may be contributed by members of the broader microbial community, for example Hyphomonadaceae SP12AHP1 and SP5AHP2, which encode a highly expanded family of novel aromatic flavin-dependent halogenases (Supplementary Figure S10AB, Additional file 1). Several of these halogenases occur in sets of 2-4 tandem repeats, suggesting family expansion by gene duplication. Based on similarity to Pfam database model PF04820 [70], these proteins are annotated as flavin-dependent tryptophan halogenases. However, their closest match among experimentally characterized enzymes is the $b r v H$ gene product from Brevundimonas sp. BAL3, at $47 \%$ amino acid identity (Supplementary Figure S10B, Additional file 1). BrvH uses free indole rather than tryptophan as a substrate, preferentially incorporating bromine over chlorine into the $\mathrm{C} 3$ position [71].
It is possible that some of the additional diversity in halogenated compounds previously observed in Lamellodysidea sponges, including brominated phenols and catechols [72, 73], arises from degradative, rather than biosynthetic pathways. The SP12AHP1 and SP5AHP2 genomes each encode a complete 3,5-dichlorocatechol degradation pathway, characteristic of bacteria that use halogenated benzoates as sole carbon and energy sources [74]. Non-halogenated benzoate degradation pathways are common in other Hyphomonadaceae, but the SP12AHP1 and SP5AHP2 genomes are unique in encoding chlorocatechol 1,2-dioxygenase, a key enzyme that cleaves chlorocatechol rings to 2,4-dichloro-cis,cismuconate, followed by spontaneous dehalogenation during further processing by muconate cycloisomerase, carboxymethylenebutenolidase, and maleylacetate reductase [75]. The closest chlorocatechol 1,2-dioxygenase match in the GenBank nr database (64\% amino acid identity) was found in Altererythrobacter marensis, a free-living coastal marine bacterium from the Alphaproteobacteria order Sphingomonadales [76]. Dichlorocatechol degradation pathways in the SP12AHP1 and SP5AHP2 genomes are clustered together in the same conserved gene order as A. marensis (Supplementary Figure S11, Additional file 1).

Plasmids containing the 3,5-dichlorocatechol degradation pathway are frequently exchanged between environmental bacteria in chloroaromatic contaminated environments (reviewed in [77]). Although the pathway is not located on a plasmid or within a genomic island in SP12AHP1, SP5APH2, or A. marensis, patchy phylogenetic distribution and order-level relationships between closest protein sequence relatives suggest historical dissemination by horizontal gene transfer (Supplementary Figure 12, Additional file 1). It is not known whether amino acid sequence similarity to experimentally characterized chlorocatechol dioxygenases might also capture activity towards brominated substrates, including PBDEs, but this seems like a reasonable hypothesis for future testing.

\section{Discussion}

Sponge-associated microbial communities have historically been classified into two broad categories, designated HMA (high microbial abundance, high diversity) and LMA (low microbial abundance, low diversity) [78]. Despite the fact that these legacy descriptions exclude situations where abundance and diversity might not be correlated, they are pervasive in the recent sponge microbiome literature, perhaps because physical quantification of microbial cell abundance in sponge tissues is technically challenging and rarely performed. High sponge seawater pumping rates have been proposed to promote aerobic, LMA communities, with lower rates 
favoring more anaerobic HMA communities [2]. These hypotheses are supported by results in the current study, where multiple samples from Lamellodysidea herbacea sponges, noted for high seawater pumping rates [3], contain a predominance of bacteria favoring aerobic rather than anaerobic metabolic pathways, with low overall community diversity.

The Lamellodysidea herbacea sponge microbiome has previously been characterized almost exclusively in terms of functional activities associated with its most dominant symbiont, Hormoscilla spongeliae. The results of this study reveal previously unreported potential metabolic activities, interactions, and community contributions of non-Hormoscilla species. Low microbial community complexity facilitated effective taxonomic binning and metagenomic assembly, producing long scaffolds enabling operon-context assessment of functional gene activities. The assembly of multiple population genomes from closely related taxa in different, independent samples helped establish core functional characteristics for novel sponge-associated groups previously known only from $16 \mathrm{~S}$ rRNA gene fragments, and encountered only rarely in other sponge species.

The GM7ARS4 and GM202ARS2 genomes, classified in a new Alphaproteobacteria order (Candidatus Methylospongiales), and SP5GCR1, representing a new Gammaproteobacteria family, are, to the best of our knowledge, the first reported potential pathogens of Lamellodysidea herbacea, although type III secretion systems may also be associated with commensal or mutualistic relationships. The unlikely combination of methylotrophy with type III secretion systems consistently shared in distantly related taxa suggests potential exploitation of a common niche opportunity. Although no close relatives of these organisms have yet been reported in other sponges, the three near-complete genomes obtained in this study should enable more effective future searches based on predicted metabolic and physiological features.

The genomes sequenced in this study show strong evidence of adaptation to their sponge-host environment. Abundant light availability in the reef habitat fuels energy generation through three different processes, using phototrophic pigments with widely varying absorption maxima: chlorophyll $a(660 \mathrm{~nm})$, bacteriochlorphyll $a(800 \mathrm{~nm})$, and proteorhodopsin $(\sim 520 \mathrm{~nm})$. UV damage protection is provided by carotenoid pigments. The possibility that photoactive pigments with differing optimal absorption spectra might provide advantages within micro-niches associated with different layers of sponge tissue would be an interesting topic for future studies.

High seawater flow rates induced by host pumping action create strong shear forces that could potentially dislodge microbiome inhabitants from sponge tissues. The expanded families of adhesive molecules and type IV pili found in many Lamellodysidea-associated bacteria would be expected to counteract these disruptive forces. Oxygen depleted by microbial respiration should be rapidly replenished from seawater flow and cyanobacterial photosynthesis, consistent with an environment that appears to favor organisms using aerobic processes for heterotrophic degradation of dissolved and particulate organics, and potentially discriminating against symbionts requiring more anaerobic conditions. Nitrogenase enzymes requiring the exclusion of oxygen were not observed. They may however be unnecessary in this environment due to a combination of host ammonia excretion and the release of fixed nitrogen from the degradation of organic compounds [2].

Previous bioenergetic analyses of microbial contributions to Lamellodysidea sponge nutrition have been limited to relatively simplistic models based on chlorophyllmediated photosynthesis by a single microorganism. Functional pathway predictions and abundance data describing the new phototrophic and heterotrophic symbionts identified in this study should allow the expansion of these models to include contributions from multiple metabolic sources, encompassing a more accurate sampling of microbiome community diversity.

Microbial community compositions in all Lamellodysidea samples were strongly correlated with host clade taxonomy, whether assessed by metagenomic read recruitment or 16S rRNA gene analysis. The persistent co-occurrence of highly conserved Rhodobacteriaceae and Rhodospirillaceae species with Hormoscilla over an 11-year time period in Guam Lamellodysidea sponges (from 2005 in [14] to 2016 in the current work) suggests the likelihood of relatively stable, long-term hostmicrobe relationships. Previous analyses of Guam Lamellodysidea sponge microbiomes [14] failed to identify many of the taxa described in the current study, but examined 100-fold fewer 16S rRNA gene sequences, and did not include any representatives of host clades Ia or Ib. Further studies will be required to understand why host clade Ia contains some taxa, especially Hyphomonadaceae and Prochloron, that are absent in other clades, as well as why photoheterotrophic Rhodobacteriaceae were relatively abundant in clades Ia, Ib, and III, but nearly absent from clade II, where they were replaced by non-photosynthetic Rhodospirillaceae.

The biological functions and effects of locally concentrated PBDEs produced by Hormoscilla bacteria are unknown. Genomes SP12AHP and SP5AHP2 from clade Ia PBDE-producing sponges encode a potential mechanism for aerobic degradation of these compounds that may enable their use as nutritional energy sources. This proposed pathway includes a modified dioxygenase ring 
cleavage mechanism related to those used by other Hyphomonaceae for breaking down non-halogenated aromatic hydrocarbons.

A large number of biologically active secondary metabolite compounds have been isolated from Lamellodysidea herbacea sponges (reviewed in [79]), but distinguishing between those synthesized by the eukaryotic host versus microbial symbionts has proved challenging, especially in the absence of an assembled host genome. The unexpected discovery that the microbiomes of Lamellodysidea herbacea clade Ia sponges contain representatives of the prolific secondary metabolite-producer genus Prochloron, in addition to Hormoscilla, provides new opportunities to identify microbial sources for both previously characterized and undiscovered natural product molecules.

\section{Conclusions}

This work has demonstrated sponge microbial community compositions to be both clade-specific and consistent over multi-year timescales. New insights have been provided into potential metabolic contributions of nondominant members of Lamellodysidea microbial communities, encompassing energy generation through anoxygenic photosynthesis, methylotrophic C-1 metabolism, and aerobic heterotrophy of organic compounds, potentially including polybrominated diphenyl ethers produced by the dominant cyanobacterium, Hormoscilla. Genomic evidence suggests that both biosynthesis and degradation of the secondary metabolome may be linked among multiple taxa within the Lamellodysidea microbiome.

The reconstruction of high-quality MAGs representing all microbial taxa at relative abundances greater than $0.1 \%$ has enabled species-specific assignment of unique metabolic features that could not have been predicted from taxonomic data alone. This information will promote more representative models of marine invertebrate microbiome contributions to host bioenergetics and a better understanding of biosynthetic and degradative pathways for secondary metabolites and halogenated compounds in sponge-associated microbiota.

\section{Methods}

\section{Sample collection and processing}

Sponge samples used in this study, shown in Supplementary Table 1, Additional file 1, were collected between 2014 and 2016 from four different coastal sites in Guam. Frozen tissue samples were processed and prepared for DNA sequencing using Illumina HiSeq pairedend technology as described previously [13, 16]. Additional PacBio sequencing reads, obtained from cyanobacterial trichome enrichments of samples GUM7 and GUM202 [13], were used in the assembly of Hormoscilla spongeliae genomes from these samples.
Sample host taxonomy was verified by amplification of ITS primer regions from bulk DNA, according to the protocol described in [80].

16S rRNA gene amplification was performed using V4-5 region primers 515F-4Y (5'-GTGYCAGCMGCCGCGGTAA) and 926R (5'-CCGYCAAT TYMTTTRAGTTT), as previously described [81]. Paired-end reads were generating using Illumina MiSeq v3 $(2 \times 300 \mathrm{bp})$ sequencing. DADA2 v 1.12 [82] was used within QIIME2 [83] to trim, denoise, merge, remove chimeras, and cluster the assembled reads, with the following program parameters: --p-trunc-len-f 250 --p-trunc-len-r 180 --p-trim-left-f 20 --p-trim-left-r 20 --p-max-ee-f 3 --p-max-ee-r 5. Taxonomic classifications were assigned based on the SILVA v132 reference database [84], supplemented with 19 near-complete $16 \mathrm{~S}$ rRNA genes obtained from targeted metagenomic assemblies in this study.

\section{Metagenomic assembly}

Illumina HiSeq 2500 reads $(2 \times 100$ or $2 \times 150 \mathrm{PE})$ from metagenomic DNA libraries were quality filtered and trimmed using Trimmomatic version 0.339 [85]. Preliminary guide assemblies were created using IDBA-UD version 1.1.117 [86] with default parameters, followed by mapping of input reads to scaffolds using the end-to-end option of Bowtie2, version 2.218 [87]. Coverage depth and percentage of singleton versus paired reads were calculated from output of the idxstats module from samtools version 0.1.191 [88]. Preliminary scaffold bins were assigned based on percent GC, nucleotide composition, coverage depth, and taxonomic classification by DarkHorse version 2.0 [89, 90], as previously described [91]. Read subsets were supplemented as necessary to include both R1 and R2 members of each constituent read pair, then re-assembled.

Second round assemblies were performed using both IDBA-UD, and, alternatively Celera Assembler version 8.3 [92], configured with merSize $=17$, utgGenomeSize $=5 \mathrm{Mb}$, and utgErrorRate $=0.01$. Second round assembly scaffolds were quality-screened by re-applying the same methods used for initial taxonomic binning (percent GC, coverage depth, and DarkHorse taxonomic classification), with an added requirement that each scaffold should contain more than $90 \%$ paired (versus singleton) reads. Final assembly completeness and contamination were assessed using CheckM version 1.07 with the default set of bacterial marker genes [24].

Hormoscilla genome assemblies have been previously reported for samples GUM202 and GUM007 using a combination of Illumina and PacBio reads from trichome-enriched preparations [13]. In the current study, PacBio reads from sample GUM007 were reassembled without Illumina read supplementation using 
CANU version 1.8 with default parameters [93], which greatly improved the GUM007 Hormoscilla assembly ( $100 \%$ versus $94.5 \%$ completeness, $1.8 \%$ versus $2.2 \%$ contamination, and 6 versus 64 contigs). This approach could not, however, be applied to the GUM202 Hormoscilla genome, because the smaller number of available PacBio reads $(31,034$ versus 180,838$)$ failed to provide sufficient coverage.

\section{Phylogenetic placement}

16S rRNA gene sequences for closely related species were obtained by blastn searches against SILVA database release 132 [94], and aligned with sequences extracted from assembled genomes using the SILVA Incremental Aligner (SINA) version 1.2.11 [95]. 16S rRNA gene trees were constructed from these alignments using FastTree version 2.1.8 [96] and visualized with FigTree version 1.4.3 [97]. Multi-locus gene trees were constructed using PhyloPhlAn version 0.99 [98]. Average amino acid identities were calculated using the online AAI-Matrix Genome-based distance matrix calculator [26].

\section{Environmental database searches}

16S rRNA genes identified in metagenome-assembled genomes (MAGs) from this study were compared to reference database sets by blastn searches, with a minimum cutoff alignment length of 360 nucleotides and maximum $e$ value of $1 \mathrm{e}-7$. Quantitative abundances of blastn matches to OTUs from samples in the Sponge Microbiome Database were obtained by querying a custom MySQL database, constructed by reformatting Supplementary files S3, S4, and S5 from [30] into the schema shown in Supplementary Figure 13, Additional file 1.

\section{Functional annotation}

All MAGs generated in this study were annotated at IMG-MER [99]. CRISPR repeat regions were identified using the MinCED (Mining CRISPRs in Environmental Datasets) program, version 0.3.0 [100]. Potentially overrepresented protein functional families were identified using Hidden Markov Models (HMMs) from the PfamA version 32 [70] and TIGRFAM release 15 [101] databases. In cases where models with overlapping functional activities matched the same target protein, only the HMM with the highest bitscore was included in quantitative tallies, so that no protein was counted more than once. Candidate viral sequences were identified using VirSorter version 1.0.5 [58] and taxonomically classified using DarkHorse version 2.0 with a custom reference database consisting of all viral sequences in the GenBank nr database as of January 2, 2019. Biosynthetic clusters encoding potential secondary metabolites were identified using AntiSMASH version 5.0 [62].

\section{Supplementary information}

Supplementary information accompanies this paper at https://doi.org/10. 1186/s40168-020-00877-y.

Additional file 1: Supplementary Figure 1. Cyanobacteria MAGS classified taxonomically. A) PhyloPhlAn multi-locus concatenated tree, with Crinalium epipsammum as an outgroup. B) 165 rRNA gene/and average amino acid identity matrix with closest database relatives. Guidelines for assigning species, genus, family, and order-level taxonomic granularity were based on $[25,26]$ for AAl and [38] for 165 rRNA gene percent nucleotide identity. Supplementary Figure 2. Bacteroidetes MAGs classified taxonomically. A) PhyloPhIAn multi-locus

concatenated tree, with SP5OBV1 as an outgroup. B) $16 \mathrm{~S}$ rRNA gene/ and average amino acid identity matrix with closest database rela-

tives. Supplementary Figure 3. Alphaproteobacteria MAGs classified taxonomically. A) PhyloPhIAn multi-locus concatenated tree, with SP5OBV1 as an outgroup. B) $16 \mathrm{~S}$ rRNA gene/and average amino acid identity matrix with closest database relatives. Supplementary Figure 4. Gammaproteobacteria MAGs classified taxonomically. A) PhyloPhIAn multi-locus concatenated tree, with SP5OBV1 as an outgroup. B) $16 \mathrm{~S}$ rRNA gene/and average amino acid identity matrix with closest database relatives. Supplementary Figure 5. Oligoflexia MAG classified taxonomically. A) PhyloPhlAn multi-locus

concatenated tree, with Oceanobaculum indicum as an outgroup. B) $16 \mathrm{~S}$ rRNA gene/and average amino acid identity matrix with closest database relatives. Supplementary Figure 6 . Relative abundance of metagenomically assembled sequences in $16 \mathrm{~S}$ rRNA gene amplicon data sets. Amplified $16 \mathrm{~S}$ rRNA gene sequences were recruited by blastn search at $97 \%$ identity to metagenomically assembled $16 \mathrm{~S}$ rRNA genes and pooled into taxonomic groups as shown in Figure 3. Relative abundances in this chart are normalized to compensate for differences in total number of 165 rRNA reads obtained for each sponge sample (see Supplementary table 2 for total numbers of cleaned, merged amplicon reads). MAGs GM102CHS1, GM7ARS4, SP5ARS3, GM102ARS1 could not be included in this table because they did not contain any sequences overlapping the amplified region of the $16 \mathrm{~S}$ rRNA gene. Supplementary Figure 7. Relative abundance of raw metagenomic reads mapping to assembled MAGS. Percentages are based on Bowtie read recruitment at 100\% identity. MAGs were pooled into taxonomic groups as shown in Figure 3. A) Relative abundances for all classified reads; B) read recruitment percentages, including unclassified reads. All percentages have normalized for number of reads per sample, but not adjusted for potential differences in genome size. Supplementary Figure 8. Host associations of Type III secretion protein YscR homologs. A) Highlighted YscR homologs from MAGs of this study are shown in the context of a phylogenetic tree annotated with host types as described in [41]. B) Position of YscR homolog in Yersinia Type III secretion structure, adapted from [103]. Supplementary Figure 9. VirSorter candidate scaffolds in holobiont assemblies. Virus taxonomic groups were obtained by DarkHorse assignment based on blastx matches to a custom database containing all viral sequences in the GenBank nr database as of January 2, 2019. Supplementary Figure 10. Flavindependent aromatic halogenases from SP12AHP1 and SP5AHP2. A) Operon structure map from IMG-MER [102] showing relative positions of tandem repeats in red. B) Phylogenetic tree of protein sequences, showing positions of putative halogenase sequences from this study relative to experimentally characterized database examples described in [71]. The tree is rooted with the bmp5 gene of Pseudoalteromonas putida [104]. PBDE pathway halogenases from Hormoscilla strains SP5, SP12, GUM102, and GUM202 clade with the bmp5 outgroup. Supplementary Figure 11. Comparison of candidate haloaromatic degradation gene neighborhoods. A) Gene neighborhood maps with halocatechol dioxygenase genes indicated in red. Maps and tables of predicted genes for B) SP12AHP1, C) SP5AHP2 and D) Altererythrobacter marensis were obtained using the automated IMG-ER annotation pipeline [102]. Supplementary Figure 12. Phylogenetic tree of halocatechol 1,2 dioxygenases. Tree shows SP12AHP1 and SP5AHP1 halocatechol 1,2-dioxygenases, highlighted in red, compared to closest GenBank nr database protein relatives. Chemically characterized 
chlorocatechol 1,2-dioxygenases are highlighted in blue. Supplementary Figure 13. MySQL schema for extracting sample metadata and abundance statistics from the Sponge Microbiome Database. Tables were populated using data in Supplementary tables 3,4, and 5 from [30]. Supplementary Table 1. Lamellodysidea sponge sampling metadata. Supplementary Table $\mathbf{2}$. Sponge holobiont and trichome metagenomic assembly statistics. Note: *GUM202 trichome enrichment reads were assembled in combination with Illumina GUM202 holobiont sequences, as previously described in [13]. Supplementary Table 3. MIMAG parameters for assembled genomes. Supplementary Table 4. Closest environmental matches from the GenBank nr database to 165 rRNA genes obtained from MAGs. GM102CHS1, GM7ARS4, SP5ARS3, GM102ARS1 are not included in this table because they did not include any 165 rRNA genes of $300 \mathrm{nt}$ or longer. Supplementary Table 5. Assembled MAG $16 \mathrm{~S}$ rRNA gene matches to Sponge Microbiome Database samples at $97 \%$ nucleotide identity. Database sequences were $99 \mathrm{bp}$ segments amplified from the V4 region of the 16S rRNA gene [30]. Sponge Microbiome Database samples with the maximum number of matches to each MAG-associated $16 \mathrm{~S}$ rRNA gene from this study were selected to determine the number and percent of matched sequences, and reported here along with host genus and geographical collection site. GM102CHS1, GM7ARS4, SP5ARS3, GM102ARS1 are not included in this table because they did not include any sequences overlapping the $\mathrm{V} 4$ region of the $16 \mathrm{~S}$ rRNA gene. Supplementary Table 6 . Tally of membrane-associated gene products in MAGs. Values for adhesive genes, signal sequences, and transporter types were tallied based on keyword searches of MAG annotations at IMG-MER [102]. Supplementary Table 7. Viral defense genes in assembled MAGs. Values were tallied based on keyword searches of MAG annotations at IMG-MER [102]. Supplementary Table 8. Biosynthetic gene clusters predicted by AntiSmash [62], supplemented with aromatic halogenases predicted by Pfam database model PF04820 [70].

\section{Abbreviations}

PBDE: Polybrominated diphenyl ether; ITS: Internal transcribed spacer; Mbp: Megabase pair; nt: Nucleotide; MAG: Metagenome-assembled genome; TCA: Tricarboxylic acid; NRPS: Non-ribosomal peptide synthase; HMM: Hidden Markov Model; PKS: Polyketide synthase

\section{Acknowledgements}

We thank our University of California, San Diego colleagues Dr. Alvaro Muñoz Plominsky for critical reading and suggestions for improvement of the manuscript and Katherine Bauman for assistance in sponge collections and DNA isolation.

\section{Authors' contributions}

S.P., V.A., B.S.M., and E.E.A. designed the study. S.P., J.M.B., A.O., and M.A.S. performed the experiments. J.B. provided the sponge samples, analytical tools, and reagents. S.P. and E.E.A wrote the manuscript with input from all authors. The authors read and approved the final manuscript.

\section{Funding}

This work was supported by grants from NSF (OCE-1837116) and NIEHS (R01ES030316 to BSM and EEA, and R00-ES026620 to V.A.).

\section{Availability of data and materials \\ Sequence datasets generated and/or analyzed during the current study (sequencing reads, genome assemblies, and amplified ITS and 16S rRNA sequences) are available in the NCBI GenBank repository under BioProject ID PRJNA320446, and the IMG-MER database [102] under genome accession numbers 2643221529, 2747842407, 2758568580, 2758568581, 2767802581, 2808606420-34, 2814122959, 2830827416, and 3300008641.}

Ethics approval and consent to participate

Not applicable

\section{Consent for publication}

Not applicable

\section{Competing interests}

The authors declare no conflicts of interest.

\section{Author details}

${ }^{1}$ Marine Biology Research Division, Scripps Institution of Oceanography, University of California, San Diego, La Jolla, CA, USA. ${ }^{2}$ Laboratory of Microbiology, Wageningen University, Wageningen, The Netherlands. ${ }^{3}$ School of Chemistry and Biochemistry and School of Biological Sciences, Georgia Institute of Technology, Atlanta, GA, USA. ${ }^{4}$ University of Guam Marine Laboratory, UoG Station, Mangilao, GU, USA. ${ }^{5}$ Center for Marine Biotechnology and Biomedicine, Scripps Institution of Oceanography, University of California, San Diego, La Jolla, CA, USA. ${ }^{6}$ Skaggs School of Pharmacy and Pharmaceutical Sciences, University of California, San Diego, La Jolla, CA, USA. 'Center for Microbiome Innovation, University of California, San Diego, La Jolla, CA, USA. ${ }^{8}$ Division of Biological Sciences, University of California, San Diego, La Jolla, CA, USA.

Received: 6 November 2019 Accepted: 28 May 2020

Published online: 23 June 2020

\section{References}

1. Weisz JB, Lindquist N, Martens CS. Do associated microbial abundances impact marine demosponge pumping rates and tissue densities? Oecologia. 2008;155(2):367-76.

2. Maldonado $M$, Ribes $M$, van Duyl FC. Nutrient fluxes through sponges: biology, budgets, and ecological implications. Advances in Sponge Science: Physiology, Chemical and Microbial Diversity, Biotechnology. 2012;62:113-82.

3. Biggerstaff A, et al. Metabolic responses of a phototrophic sponge to sedimentation supports transitions to sponge-dominated reefs. Sci Rep. 2017:7(1):2725.

4. Taylor MW, et al. Soaking it up: the complex lives of marine sponges and their microbial associates. ISME J. 2007;1 (3):187-90.

5. Pita $L$, et al. The sponge holobiont in a changing ocean: from microbes to ecosystems. Microbiome. 2018 Mar 9;6(1):46.

6. Webster NS, Thomas T. The sponge hologenome. MBio. 2016;7(2):e00135-16.

7. Bercerro MA, P.V., Effects of depth and light on secondary metabolites and cyanobacterial symbionts of the sponge Dysidea granulosa. Mar Ecol Prog Ser, 2004. 280: p. 115-128.

8. Thacker RW. Impacts of shading on sponge-Cyanobacteria symbioses: a comparison between host-specific and generalist associations. Integrative and Comparative Biology. 2005;45(2):369-76.

9. Hinde R, Pironet F, Borowitzka MA. Isolation of Oscillatoria-Spongeliae, the filamentous cyanobacterial symbiont of the marine sponge DysideaHerbacea. Marine Biology. 1994;119(1):99-104.

10. Usher KM. The ecology and phylogeny of cyanobacterial symbionts in sponges. Marine Ecology-an Evolutionary Perspective. 2008;29(2):178-92.

11. Freeman CJ, et al. Quality or quantity: is nutrient transfer driven more by symbiont identity and productivity than by symbiont abundance? ISME J. 2013;7(6):1116-25.

12. Berthold RJ, Borowitzka MA, Mackay MA. The ultrastructure of OscillatoriaSpongeliae, the blue-green-algal endosymbiont of the sponge DysideaHerbacea. Phycologia. 1982;21(3):327-35.

13. Schorn MA, et al. Comparative genomics of cyanobacterial symbionts reveals distinct, specialized metabolism in tropical Dysideidae sponges. MBio. 2019;10(3):e00821-19.

14. Ridley CP, John Faulkner D, Haygood MG. Investigation of Oscillatoria spongeliae-dominated bacterial communities in four dictyoceratid sponges. Appl Environ Microbiol. 2005;71(11):7366-75

15. Ridley CP, et al. Speciation and biosynthetic variation in four dictyoceratid sponges and their cyanobacterial symbiont. Oscillatoria spongeliae. Chem Biol. 2005;12(3):397-406.

16. Agarwal $\mathrm{V}$, et al. Metagenomic discovery of polybrominated diphenyl ether biosynthesis by marine sponges. Nat Chem Biol. 2017;13(5):537-43.

17. Unson MD, Holland ND, Faulkner DJ. A brominated secondary metabolite synthesized by the cyanobacterial symbiont of a marine sponge and accumulation of the crystalline metabolite in the sponge tissue. Marine Biology. 1994;119(1):1-11.

18. Hanif $\mathrm{N}$, et al. Polybrominated diphenyl ethers from the Indonesian sponge Lamellodysidea herbacea. J Nat Prod. 2007;70(3):432-5. 
19. Liu $\mathrm{H}$, et al. Polybrominated diphenyl ethers: structure determination and trends in antibacterial activity. J Nat Prod. 2016;79(7):1872-6.

20. Sharma GM, Vig B. Studies on antimicrobial substances of sponges. vi. structures of 2 antibacterial substances isolated from marine sponge Dysidea-Herbacea. Tetrahedron Letters. 1972;17:1715-8.

21. Sun $\mathrm{S}$, et al. Polybrominated diphenyl ethers with potent and broad spectrum antimicrobial activity from the marine sponge Dysidea. Bioorg Med Chem Lett. 2015;25(10):2181-3.

22. Becerro MA, et al. Biogeography of sponge chemical ecology: comparisons of tropical and temperate defenses. Oecologia. 2003;135(1):91-101.

23. Bowers RM, et al. Minimum information about a single amplified genome (MISAG) and a metagenome-assembled genome (MIMAG) of bacteria and archaea. Nat Biotechnol. 2017;35(8):725-31.

24. Parks DH, et al. CheckM: assessing the quality of microbial genomes recovered from isolates, single cells, and metagenomes. Genome Res. 2015; 25(7):1043-55

25. Rodriguez-R LM, Konstantinos T. Konstantinidis, Bypassing cultivation to identify bacterial species. Microbe. 2014;9(3):111-8.

26. Rodriguez-R L, Konstantinidis K. The enveomics collection: a toolbox for specialized analyses of microbial genomes and metagenomes. Peer」 Preprints. 2016:4:e1900v1.

27. Lewin, R.A. and L. Cheng, Prochloron: a microbial enigma. 1989, New York: Chapman and Hall. xiii, 129 p., 26 p. of plates.

28. Cheng L, Lewin RA. Prochloron on synaptula. Bulletin of Marine Science. 1984;35(1):95-8.

29. Parry DL. Prochloron on the Sponge Aplysilla Sp. Bulletin of Marine Science. 1986;38(2):388-90.

30. Thomas T, et al. Diversity, structure and convergent evolution of the global sponge microbiome. Nat Commun. 2016;7:11870.

31. Moitinho-Silva L, et al. The sponge microbiome project. Gigascience. 2017; 6(10):1-7.

32. Alain K, et al. Ekhidna lutea gen. nov., sp. nov., a member of the phylum Bacteroidetes isolated from the South East Pacific Ocean. Int I Syst Evol Microbiol. 2010;60(Pt 12):2972-8.

33. Liu Y, et al. Nioella sediminis $\mathrm{sp}$. nov., isolated from surface sediment and emended description of the genus Nioella. Int J Syst Evol Microbiol. 2017; 67(5):1271-4.

34. Rajasabapathy $R$, et al. Nioella nitratireducens gen. nov., sp. nov., a nove member of the family Rhodobacteraceae isolated from Azorean Island. Antonie Van Leeuwenhoek. 2015;107(2):589-95.

35. Wisniewski-Dye $F$, et al. Azospirillum genomes reveal transition of bacteria from aquatic to terrestrial environments. PLoS Genet. 2011;7(12):e1002430.

36. Alain K, et al. Hellea balneolensis gen. nov., sp. nov., a prosthecate alphaproteobacterium from the Mediterranean Sea. Int J Syst Evol Microbiol. 2008;58(Pt 11):2511-9.

37. Hahn MW, et al. Silvanigrella aquatica gen. nov., sp. nov., isolated from a freshwater lake, description of Silvanigrellaceae fam. nov. and Silvanigrellales ord. nov., reclassification of the order Bdellovibrionales in the class Oligoflexia, reclassification of the families Bacteriovoracaceae and Halobacteriovoraceae in the new order Bacteriovoracales ord. nov., and reclassification of the family Pseudobacteriovoracaceae in the order Oligoflexales. Int J Syst Evol Microbiol. 2017;67(8):2555-68.

38. Yarza $\mathrm{P}$, et al. Uniting the classification of cultured and uncultured bacteria and archaea using 16S rRNA gene sequences. Nat Rev Microbiol. 2014;12(9): $635-45$.

39. Martinez-Gomez NC, Nguyen S, Lidstrom ME. Elucidation of the role of the methylene-tetrahydromethanopterin dehydrogenase MtdA in the tetrahydromethanopterin-dependent oxidation pathway in Methylobacterium extorquens AM1. J Bacteriol. 2013;195(10):2359-67.

40. Gurung JM, et al. Heterologous complementation studies with the Ysc X and YscY protein families reveals a specificity for Yersinia pseudotuberculosis type III secretion. Front Cell Infect Microbiol. 2018;8:80.

41. Nazir R, et al. The ecological role of type three secretion systems in the interaction of bacteria with fungi in soil and related habitats is diverse and context-dependent. Front Microbiol. 2017:8:38

42. Klasson L, Andersson SG. Evolution of minimal-gene-sets in host-dependent bacteria. Trends Microbiol. 2004;12(1):37-43.

43. Moran NA, McCutcheon JP, Nakabachi A. Genomics and evolution of heritable bacterial symbionts. Annu Rev Genet. 2008;42:165-90.

44. Moya A, et al. Learning how to live together: genomic insights into prokaryote-animal symbioses. Nat Rev Genet. 2008;9(3):218-29.
45. Voget S, et al. Adaptation of an abundant Roseobacter RCA organism to pelagic systems revealed by genomic and transcriptomic analyses. ISME J. 2015:9(2):371-84.

46. Gonzalez JM, et al. Genomics of the proteorhodopsin-containing marine flavobacterium Dokdonia sp. strain MED134. Appl Environ Microbiol. 2011; 77(24):8676-86

47. McBride MJ, Nakane D. Flavobacterium gliding motility and the type IX secretion system. Curr Opin Microbiol. 2015;28:72-7.

48. Johnston JJ, Shrivastava A, McBride MJ. Untangling Flavobacterium johnsoniae gliding motility and protein secretion. J Bacteriol. 2018:200(2).

49. Li N, et al. The type IX secretion system is required for virulence of the fish pathogen Flavobacterium columnare. Appl Environ Microbiol. 2017:83(23).

50. Veith PD, et al. Type IX secretion: the generation of bacterial cell surface coatings involved in virulence, gliding motility and the degradation of complex biopolymers. Mol Microbiol. 2017;106(1):35-53.

51. Lasica AM, et al. The type IX secretion system (T9SS): highlights and recent insights into its structure and function. Front Cell Infect Microbiol. 2017:7:215.

52. Fraiberg $M$, et al. Discovery and characterization of cadherin domains in Saccharophagus degradans 2-40. Journal of Bacteriology. 2010;192(4):1066-74.

53. Abraham W, Rohde M. The family Hyphomonadaceae, in The prokaryotes Alphaproteobacteria and Betaproteobacteria, Rosenberg E, et al., Editors. 2014. Springer: Berlin.

54. Tully BJ, Graham ED, Heidelberg JF. The reconstruction of 2,631 draft metagenome-assembled genomes from the global oceans. Sci Data. 2018;5: 170203.

55. Sockett RE. Predatory lifestyle of Bdellovibrio bacteriovorus. Annu Rev Microbiol. 2009;63:523-39.

56. Yao J, Rock CO. How bacterial pathogens eat host lipids: implications for the development of fatty acid synthesis therapeutics. J Biol Chem. 2015; 290(10):5940-6.

57. Pascelli $C$, et al. Morphological characterization of virus-like particles in coral reef sponges. PeerJ. 2018;6:e5625.

58. Roux S, et al. VirSorter: mining viral signal from microbial genomic data. PeerJ. 2015:3:e985.

59. Brussow, H., C. Canchaya, and W.D. Hardt, Phages and the evolution of bacterial pathogens: from genomic rearrangements to lysogenic conversion. Microbiol Mol Biol Rev, 2004. 68(3): p. 560-602, table of contents.

60. Seweryn $P$, et al. Structural insights into the bacterial carbon-phosphorus lyase machinery. Nature. 2015;525(7567):68-72.

61. Martinez A, et al. Metatranscriptomic and functional metagenomic analysis of methylphosphonate utilization by marine bacteria. Front Microbiol. 2013;4:340.

62. Blin $\mathrm{K}$, et al. antiSMASH 5.0: updates to the secondary metabolite genome mining pipeline. Nucleic Acids Res. 2019;47(W1):W81-7.

63. Donia MS, et al. Complex microbiome underlying secondary and primary metabolism in the tunicate-Prochloron symbiosis. Proc Natl Acad Sci U S A. 2011;108(51):E1423-32.

64. Schmidt EW, et al. Patellamide $\mathrm{A}$ and $\mathrm{C}$ biosynthesis by a microcin-like pathway in Prochloron didemni, the cyanobacterial symbiont of Lissoclinum patella. Proc Natl Acad Sci U S A. 2005:102(20):7315-20.

65. Enisoglu-Atalay $V$, et al. Chemical and molecular characterization of metabolites from Flavobacterium sp. PLoS One. 2018;13(10):e0205817.

66. Moore BS, et al. Plant-like biosynthetic pathways in bacteria: from benzoic acid to chalcone. J Nat Prod. 2002:65(12):1956-62.

67. Xiang L, Moore BS. Biochemical characterization of a prokaryotic phenylalanine ammonia lyase. J Bacteriol. 2005;187(12):4286-9.

68. Reiter, S., et al., Characterization of an orphan type III polyketide synthase conserved in uncultivated 'Entotheonella' sponge symbionts Chembiochem, 2019 Aug 20 (Epub ahead of print).

69. Agarwal $V$, et al. Complexity of naturally produced polybrominated diphenyl ethers revealed via mass spectrometry. Environmental Science \& Technology. 2015;49(3):1339-46.

70. Finn RD, et al. The Pfam protein families database: towards a more sustainable future. Nucleic Acids Res. 2016:44(D1):D279-85.

71. Neubauer PR, et al. A flavin-dependent halogenase from metagenomic analysis prefers bromination over chlorination. PLoS One. 2018;13(5): e0196797.

72. Agarwal V, Moore BS. Enzymatic synthesis of polybrominated dioxins from the marine environment. ACS Chem Biol. 2014:9(9):1980-4.

73. Fu XO, et al. Enzyme-inhibitors - new and known polybrominated phenols and diphenyl ethers from 4 Indo-Pacific Dysidea sponges. Journal of Natural Products. 1995;58(9):1384-91. 
74. Miyazaki $R$, et al. Comparative genome analysis of Pseudomonas knackmussii B13, the first bacterium known to degrade chloroaromatic compounds. Environ Microbiol. 2015;17(1):91-104.

75. Schwien U, et al. Degradation of chlorosubstituted aromatic-compounds by Pseudomonas sp strain-B13 - fate of 3,5-dichlorocatechol. Archives of Microbiology. 1988;150(1):78-84.

76. Seo SH, Lee SD. Altererythrobacter marensis sp. nov., isolated from seawater. Int J Syst Evol Microbiol. 2010;60(Pt 2):307-11.

77. Phale PS, Shah BA, Malhotra H. Variability in assembly of degradation operons for naphthalene and its derivative, carbaryl, suggests mobilization through horizontal gene transfer. Genes (Basel). 2019:10(8).

78. Hentschel $U$, et al. Microbial diversity of marine sponges. Prog Mol Subcell Biol. 2003:37:59-88.

79. Huang IS, Zimba PV. Cyanobacterial bioactive metabolites-a review of their chemistry and biology. Harmful Algae. 2019:86:139-209.

80. Thacker RW, S.S., Host specificity of the symbiotic cyanobacterium Oscillatoria spongeliae in marine sponges, Dysidea spp. Marine Biology, 2003. 142: p. 643-648.

81. Parada AE, Needham DM, Fuhrman JA. Every base matters: assessing small subunit rRNA primers for marine microbiomes with mock communities, time series and global field samples. Environ Microbiol. 2016;18(5):1403-14.

82. Callahan BJ, et al. DADA2: High-resolution sample inference from Illumina amplicon data. Nat Methods. 2016;13(7):581-3.

83. Bolyen $\mathrm{E}$, et al. Reproducible, interactive, scalable and extensible microbiome data science using QIIME 2. Nat Biotechnol. 2019;37(8):852-7.

84. Yilmaz P, Parfrey LW, Yarza P, Gerken J, Pruesse E, Quast C, Schweer T, Peplies J, Ludwig W, Glöckner FO (2014) The SILVA and "All-species Living. Tree Project (LTP)" taxonomic frameworks. Acids Res. 42:D643-D648.

85. Bolger AM, Lohse M, Usadel B. Trimmomatic: a flexible trimmer for Illumina sequence data. Bioinformatics. 2014:30(15):2114-20.

86. Peng $Y$, et al. IDBA-UD: a de novo assembler for single-cell and metagenomic sequencing data with highly uneven depth. Bioinformatics. 2012;28(11):1420-8.

87. Langmead B, Salzberg SL. Fast gapped-read alignment with Bowtie 2. Nat Methods. 2012;9(4):357-9.

88. Li H, et al. The sequence alignment/map format and SAMtools. Bioinformatics. 2009;25(16):2078-9.

89. Podell, S. DarkHorse2 GitHub Repository. 2017; Available from: https:// github.com/spodell/Darkhorse2.

90. Podell S, Gaasterland T. DarkHorse: a method for genome-wide prediction of horizontal gene transfer. Genome Biol. 2007;8(2):R16.

91. Podell $\mathrm{S}$, et al. Assembly-driven community genomics of a hypersaline microbial ecosystem. PLoS One. 2013;8(4):e61692.

92. Myers EW, et al. A whole-genome assembly of Drosophila. Science. 2000; 287(5461):2196-204

93. Koren S, et al. Canu: scalable and accurate long-read assembly via adaptive k-mer weighting and repeat separation. Genome Res. 2017;27(5):722-36.

94. Quast C, et al. The SILVA ribosomal RNA gene database project: improved data processing and web-based tools. Nucleic Acids Res. 2013:41(Database issue):D590-6.

95. Pruesse E, Peplies J, Glockner FO. SINA: accurate high-throughput multiple sequence alignment of ribosomal RNA genes. Bioinformatics. 2012;28(14):1823-9.

96. Price MN, Dehal PS, Arkin AP. FastTree 2--approximately maximumlikelihood trees for large alignments. PLoS One. 2010;5(3):e9490.

97. Rambaut, A. FigTree, version 1.4.3. In: Molecular evolytion phylogenetics and epidemiology. 2016; Available from: http://tree.bio.ed.ac.uk/software/figtree/ Accessed October, 2018

98. Segata N, et al. PhyloPhIAn is a new method for improved phylogenetic and taxonomic placement of microbes. Nat Commun. 2013;4:2304.

99. Markowitz VM, et al. IMG/M: the integrated metagenome data management and comparative analysis system. Nucleic Acids Res. 2012;40(Database issue): D123-9.

100. Bland C, et al. CRISPR recognition tool (CRT): a tool for automatic detection of clustered regularly interspaced palindromic repeats. BMC Bioinformatics. 2007;8:209.

101. Haft DH, et al. TIGRFAMs and genome properties in 2013. Nucleic Acids Res. 2013;41(Database issue):D387-95

102. Chen IA, et al. IMG/M: integrated genome and metagenome comparative data analysis system. Nucleic Acids Res. 2017;45(D1):D507-16.

103. Dewoody RS, Merritt PM, Marketon MM. Regulation of the Yersinia type II secretion system: traffic control. Front Cell Infect Microbiol. 2013;3:4.
104. Agarwal V, et al. Biosynthesis of polybrominated aromatic organic compounds by marine bacteria. Nat Chem Biol. 2014;10(8):640-7.

\section{Publisher's Note}

Springer Nature remains neutral with regard to jurisdictional claims in published maps and institutional affiliations.
Ready to submit your research? Choose BMC and benefit from:

- fast, convenient online submission

- thorough peer review by experienced researchers in your field

- rapid publication on acceptance

- support for research data, including large and complex data types

- gold Open Access which fosters wider collaboration and increased citations

- maximum visibility for your research: over $100 \mathrm{M}$ website views per year

At BMC, research is always in progress.

Learn more biomedcentral.com/submissions 